\title{
Exploitation assessment of geothermal energy from African Great Rift Valley
}

\author{
N. Bonechi, D. Fiaschi*, G. Manfrida, L. Talluri, C. Zuffi \\ ${ }^{a}$ Università degli Studi di Firenze, Dipartimento di Ingegneria Industriale, Viale Morgagni 40-44, 50134, Firenze \\ ${ }^{*}$ Corresponding author mail: daniele.fiaschi@unifi.it
}

\begin{abstract}
Countries that are in the Great Rift Valley have one of the lowest average annual electricity consumption per capita in Africa with a value of $164 \mathrm{kWh}$ per inhabitant. Furthermore, the electrification rate is $34 \%$ that is more than fifty percentage points below the world average, which is around $86 \%$.

One possible solution to improve the electrification rate is to properly exploit the energy resources present in the territory. One of the most significant energy sources of this region is certainly geothermal energy which has a potential of about $15 \mathrm{GWe}$, mostly concentrated in Ethiopia. Furthermore, it is possible to find the resource in a wide temperature range, not only to produce electricity, which, nonetheless, has a very limited exploitation rate, as only $900 \mathrm{MWe}$ are installed between Kenya and Ethiopia, but also for direct use.

In this study, two geothermal power plants for two different geothermal sites, Corbetti and Arus-Bogoria, respectively in Ethiopia and Kenya, have been hypothesized after analyzing the resource potential. For the first, which has been estimated to be of high enthalpy $\left(\sim 300^{\circ} \mathrm{C}\right)$, a flash plant configuration was assumed, and the estimated energy production potential was found to be around 50-100 MWe. While for the second, at medium enthalpy $\left(\mathrm{T}<200^{\circ} \mathrm{C}\right)$, the use of a binary cycle plant was assumed with an obtained production of about $20 \mathrm{MW}$ of electricity. Finally, the possibility of geothermal water exploitation for greenhouse heating, drying of agricultural products, civil sanitary uses, recreational uses (spa), or for industrial purposes has been assessed.
\end{abstract}

\section{African continent and Rift Valley}

Africa has many available energy resources, but a lack of adequate capacity and knowledge limits their use and exploitation. It has the lowest per capita energy consumption in the world: $16 \%$ of the world's population resides on the continent (about 1.2 billion out of 7.35 billion) and consumes $3.3 \%$ of global primary energy [1].

In Africa, there is a very high level of renewable energy available, the solar potential is enormous (10 TW), hydroelectricity and wind power are also widely present ( $350 \mathrm{GW}$ and $110 \mathrm{GW}$ respectively), while geothermal energy is less available than the other renewables but still exhibits a significant share $(15 \mathrm{GW})$ [2]. Almost all the geothermal resource is concentrated in the eastern part of the continent, more precisely around the Great Rift Valley. The countries that lie along the tectonic trench and thus, host geothermal resources are Burundi, Comoros, Congo (DRC), Eritrea, Ethiopia, Kenya, Malawi,

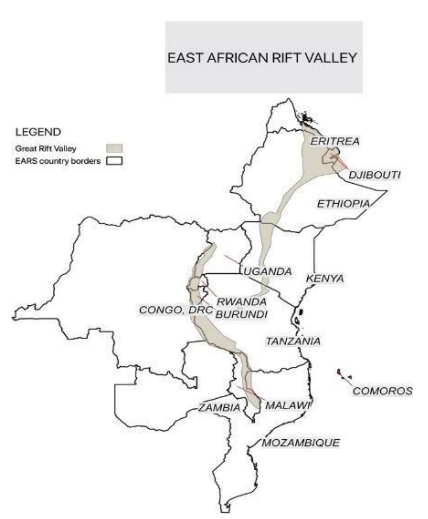

Fig. 1. EARS country Mozambique, Rwanda, Tanzania, Uganda, Zambia.

\section{Location and temperature of geothermal sites}

The geological context of East African countries supports the presence of geothermal reservoirs, these countries are in a tectonically active zone with widespread crust and volcanic activity. The geothermal resource in the region can be found both at very high enthalpy, such as the Olkaria geothermal area in Kenya, where a total of around $700 \mathrm{MW}$ have already been installed, with fluid temperature exceeding $350^{\circ} \mathrm{C}$, and at lowmedium enthalpy, with temperatures even below $100^{\circ} \mathrm{C}$.

Since there is the possibility of having low-enthalpy resources, it is worth highlighting that the exploitation of this resource can be done through its direct use, which can help to significantly reduce the use of fossil fuels and electricity in some end-use sectors. 
In the East African Rift region, direct-use applications include heating of greenhouses and aquaculture; the drying of agricultural products such as cereals, fruits, and vegetables; meat and fish processing; and cooling/refrigeration. In addition, direct-use applications can contribute to climate adaptation. For example, the use of geothermal in greenhouse heating, aquaculture, and other agricultural practices could have a positive impact on food security and could improve incomes, thus helping to address extreme poverty in rural communities. In addition, geothermal energy can provide drinking water through desalination or steam condensation on the surface to overcome water shortages. [2]

The map on the right shows the East African region and highlights the different temperature levels of geothermal wells. The temperature study focused mostly on 5 of the 13 states in this area (i.e., Kenya, Ethiopia,

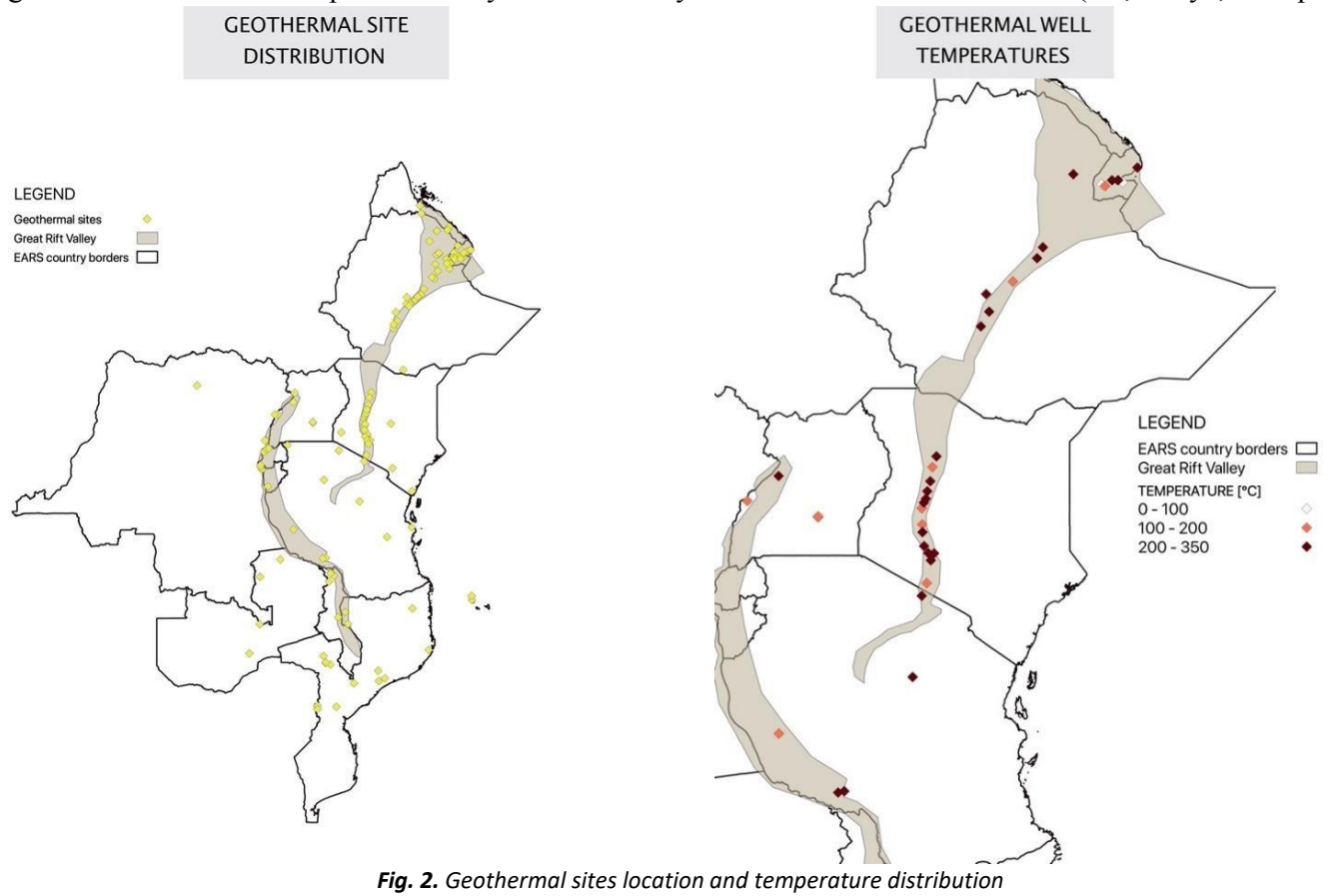

Uganda, Tanzania, Djibouti) because it was not possible to find specific and detailed data for the remaining counties. It should also be pointed out that even for countries studied some data are vacant and very often from outdated analyses [3]. As can be seen from Figure 2, high and medium enthalpy wells prevail in this area, so this allows exploitation of the resource to produce electricity.

\section{Population distribution and energy demand}

This work has been developed in several steps in order to understand which geothermal sites are the most productive and where it makes sense to invest in the construction of geothermal power plants, considering the distribution of population and energy demand.

So, the first step is a demographic study to investigate where there is the greatest concentration of human settlements. This has not been carried out with a forecast in the years for lack of data, the data concerning OpenStreetMap of the year 2020 have been taken. Then the focus became the energy demand, so identify which area was the highest with electricity consumption. In this step is necessary to divide populated places into two different classes: urban-town and country-village, in which the energy consumption of the first is greater than the second one respectively [3]. After that, it is possible to realize population density and energy demand maps using QGIS 3.10 (quantum geographic information system) software. That system works with vectorial data that can be downloaded from dedicated websites via data formats known as shapefile [4]. Vector data are recorded as a series of coordinate sets [x;y] within the computer's memory. These are used to represent points, lines, and polygons providing a way to describe the real world of objects within a GIS environment. Each of these elements owns attributes both qualitative, for example defining whether a point data is a village 
or a town, and quantitative, then express the number of people. Here are two examples of the maps created using the above software: Kenya's population distribution map and Ethiopia's energy demand map are shown. With this information, along with the geothermal site's temperatures, it was possible to forecast the most suitable areas for hosting a geothermal plant, with possible exploitation of the resource.
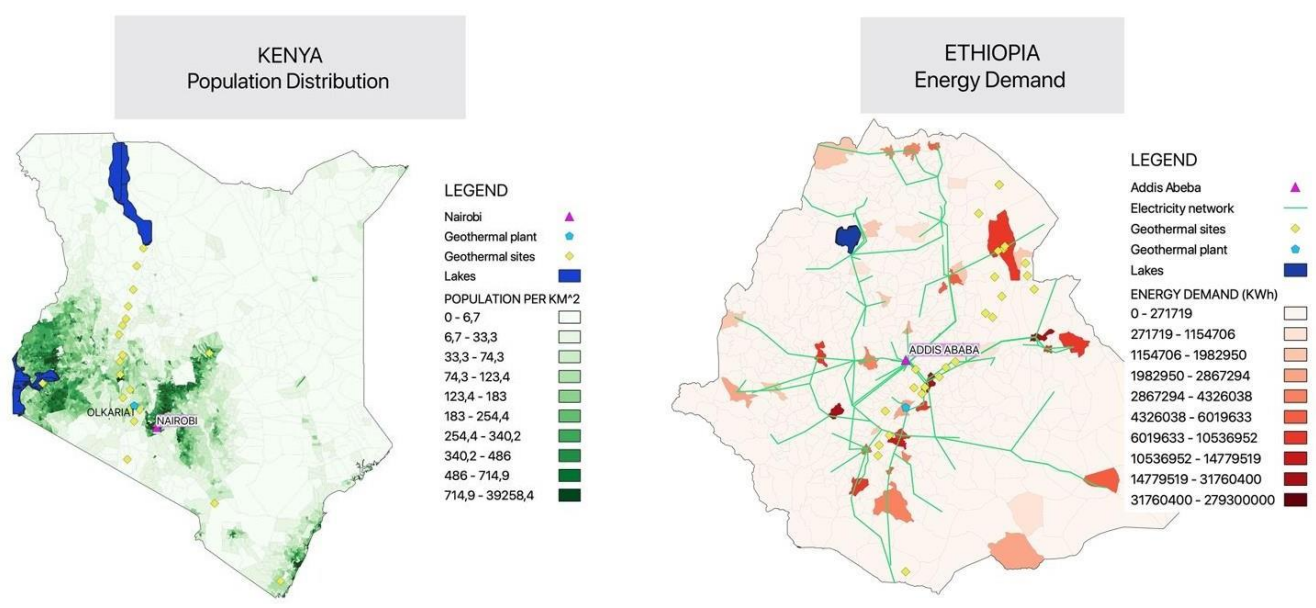

Fig. 3. Kenya's population distribution \& Ethiopia's energy demand

\section{Geothermal power plant}

Possible exploitation ways were assumed for two geothermal areas, one of high enthalpy, and one of lowmedium enthalpy.

In Ethiopia was chosen the Corbetti geothermal site. It is located about $250 \mathrm{~km}$ south of the capital Addis Ababa, in a relatively populated area and consequently also with high energy demand.

Shallow wells have already been drilled in the area, the depths reached range from 90 to 180 meters, and the temperature recorded has been around $95-100^{\circ} \mathrm{C}$. These temperatures could already be considered good for exploitation through a binary system or for direct use. Based on the geothermometric analysis of carbon dioxide, it was predicted that this geothermal area hosts a high enthalpy reservoir with temperatures of approximately $300^{\circ} \mathrm{C}$. 
Regarding the electricity network, the figure above shows that a significant power transmission of $130 \mathrm{kV}$ passes only $15 \mathrm{~km}$ far from the site and connects it to the southern part of the country. [3]

According to the high-temperature forecasts, the realization of a conventional flash technology plant is

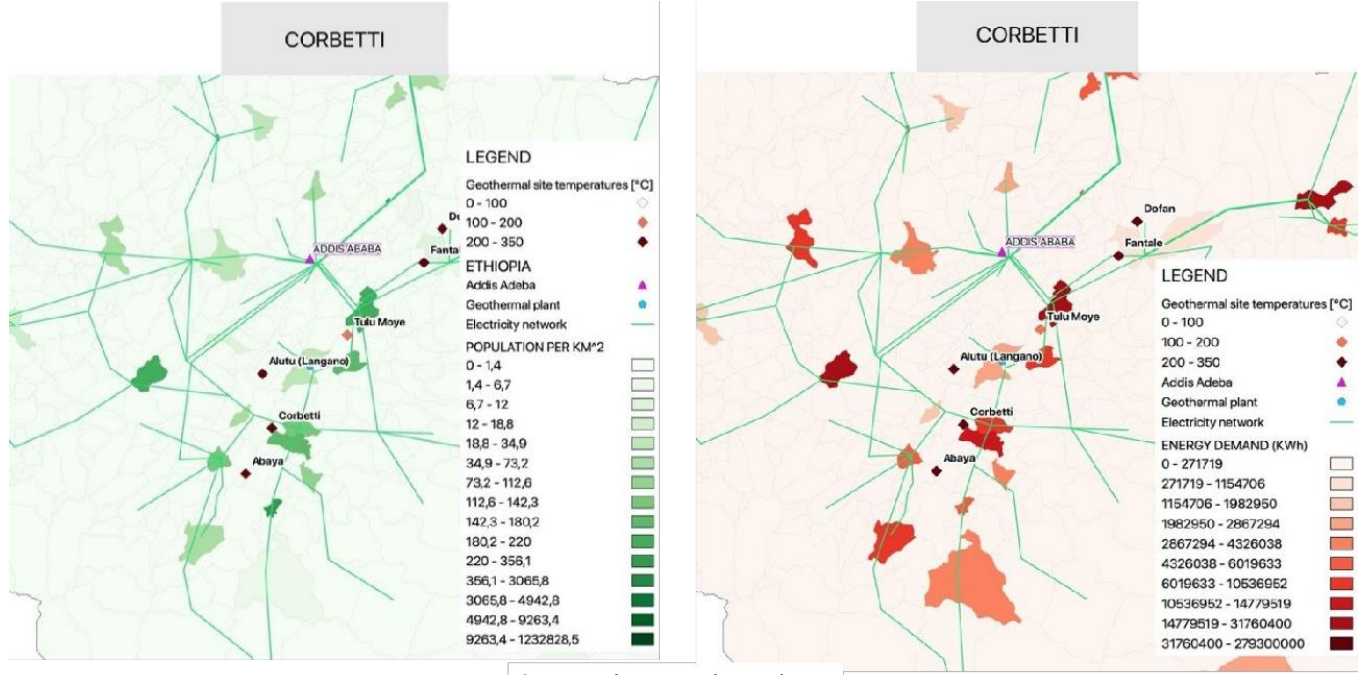

Fig. 4. Corbetti geothermal site

conceivable. Thinking about the realization of a plant of this type surely needs a chemical analysis regarding the geothermal fluid. In fact, it will be fundamental to establish if the NCG emissions are excessive and therefore need a treatment of the exhausted vapors. Under these assumptions, the geothermal resource at the Corbetti site was estimated to be able to generate an energy potential of $1000 \mathrm{MWe}$ [5]. To date, the Corbetti geothermal power project has been developed, in the Corbetti caldera region. Drilling and exploration activities began as early as 2014. The project consists of two phases: first to build a $50 \mathrm{MWe}$ plant and later $100 \mathrm{MWe}$ [6].
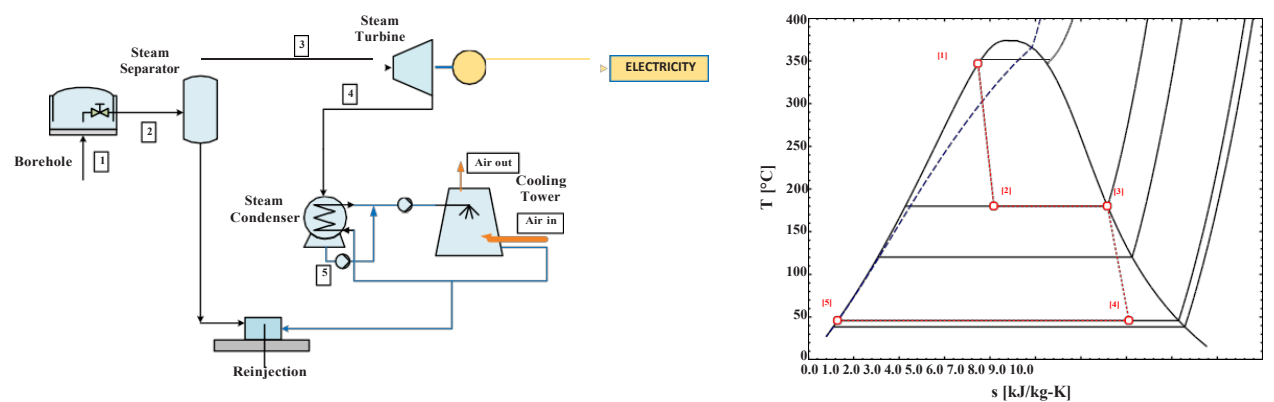

Fig. 5. Single flash geothermal plant: schematic plant and T-s diagram [11]

The second site analyzed was Arus-Bogoria in Kenya. 


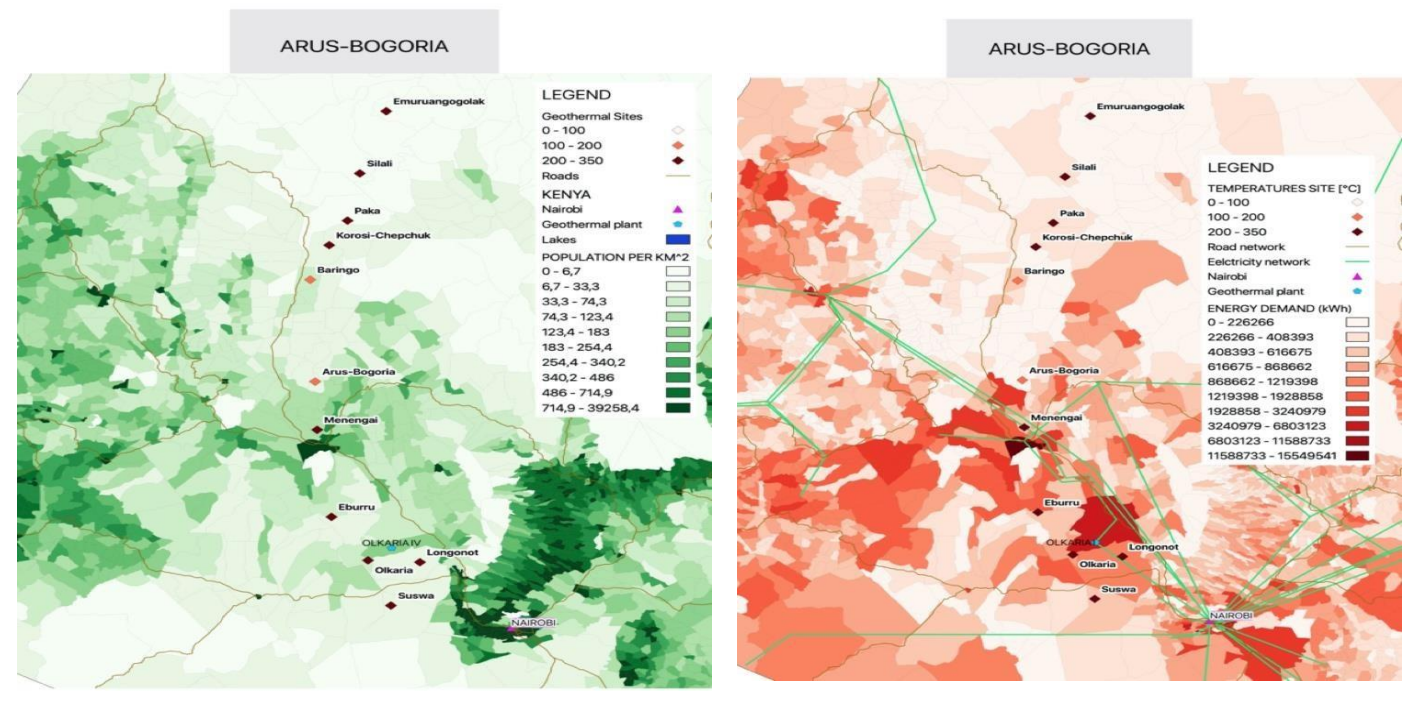

Fig. 6. Arus-Bogoria geothermal site

The site is in the south-central part of the country, a widely populated and energy-intensive area. The development phase in this area is still in the beginning; it has been recognized as a potential location for geothermal activities, but pre-feasibility studies have not yet been completed. Geothermal manifestations are abundant in the area around Lake Bogoria and fluid geothermometric studies indicate moderate subsurface temperatures of around $145-200^{\circ} \mathrm{C}$, while temperatures in Arus are below $170-200^{\circ} \mathrm{C}$. The geothermal reservoir is estimated to be small, discrete, and confined in the neighboring fault zone. However, through the installation of an ORC binary plant, a geothermal potential for the area of more than 20 MWe has been foreseen [3].
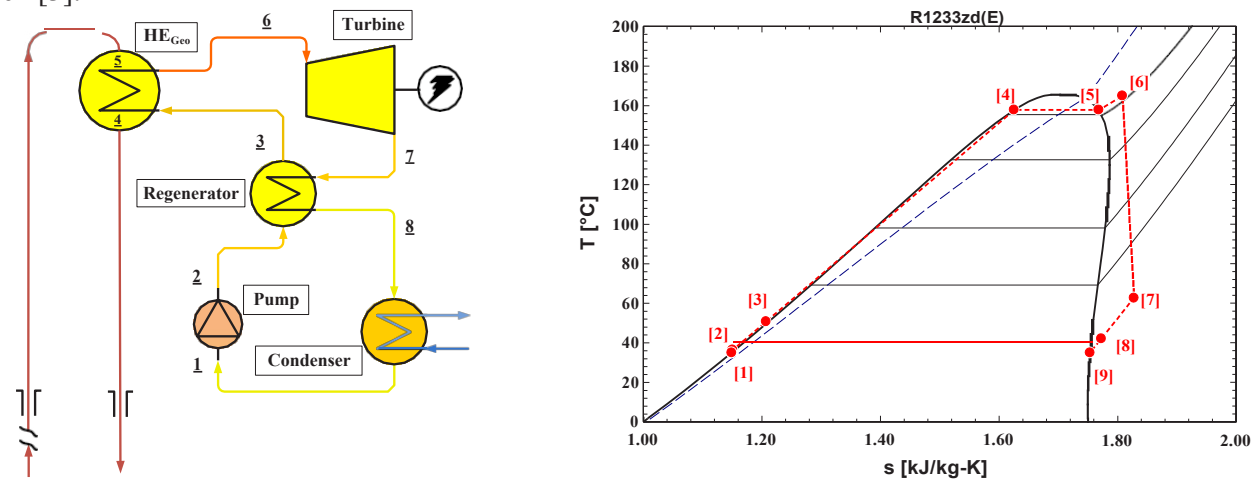

Fig. 7. Organic Rankine Cycle: plant scheme and T-s diagram for the organic fluid [11]

\section{Direct and other use of geothermal fluid}

The peculiarity of geothermal energy is that if the heat source is not hot enough to produce electricity, it is possible to use geothermal fluid directly and then exploit its heat in other systems. Otherwise, an even more efficient solution is to use the fluid leaving the power plant and exploit that heat for other purposes. Conventionally, a geothermal power plant reinjects the geothermal fluid into the ground once the fluid has been extracted and converted from thermal energy to electrical energy. To optimize the use of this fluid, it is possible to think of further uses of the power plant's fluid output, called geothermal brine, before reinjecting 
it. Moreover, it is very important to understand that the useful brine energy depends on its temperature in the last section of the heat exchanger. Usually, heat exchangers are installed at the reinjection wells to maximise energy extraction and minimise brine transportation and so reduce the scaling problems.

There is a great potential for both electricity generation and direct utilisation of geothermal energy in the Kenya Rift Valley. Direct application of brine use, show potential in:

- Balneology and spa. At Olkaria and Eburru, it is possible to use brine heat like a by-product of the geothermal power plant to provide hot water for residential houses and hotels. Near Lake Bogoria is utilizing hot water to heat a spar pool [7].

- Drying agricultural product. Geothermal energy as an alternative to solar energy to dry vegetables, fruits, and other cereals. An example is in Eburru, where farmers use geothermal energy to dry pyrethrum flowers [8].

- Greenhouse heating. in Naivasha, farmers use geothermal energy to heat 50 hectares of the greenhouse, and this involves a drastic reduction in operating cost [9].

- Industrial processes. it would be worthwhile to consider the possibility of using geothermal energy in industrial processes like the sugar cane industry, production of alcohol, wood industry, liquid $\mathrm{CO}_{2}$ mining [10].

- Residential heating or cooling with Heat pumps (GHP). the annual temperature ranges are not extreme in Kenya, so GHP's would be used to cool and heat residential houses in the cooler months [10].

Geothermal applications are many and the fields of application are very varied. The energy that can be obtained, in addition to the production of electricity, is in the form of heat and certainly, there is already a large market for this type of exploitation like industrial activities, agricultural, and other human use.

\section{Conclusions}

The aim of this research was to understand how geothermal resources are distributed, to analyze the population density and energy demand of the countries located in the Great Rift Valley, and finally to suggest a possible way of development and exploitation.

After a brief overview of the African continent and the countries examined, QGIS, the software that has made it possible to create geographical maps, was introduced. Population and energy maps were made for all the countries in the Great Rift Valley, however, as temperature data were available for only a few countries, five of the thirteen states were analyzed in more detail, and only for two states, Ethiopia and Kenya were considered for possible exploitation.

The Corbetti power plant can generate a capacity of about 50-100 MWe, while the Arus-Bogoria power plant could produce more than 20 MWe $[3,6]$.

It is possible to hypothesize various scenarios in which the low-enthalpy geothermal fluid is used directly, or the use of geothermal brine coming out of power plants to obtain heat. The market for these applications is very broad, and so is the geothermal resource in the Great Rift Valley.

Once the obstacles imposed by the lack of awareness of the benefits and advantages of direct geothermal use, limited public founding, and the lack of a skilled workforce have been overcome, the development of geothermal plants would succeed in establishing themselves and the geothermal resource as the region's primary energy source, enabling good economic growth and making up for the widespread poverty affecting a large part of the population.

\section{References}

1. Environmental Pulse Institute (EPI) U.S.A., 2017. Atlas of Africa Energy Resource. United Nation Environment Programme.

2. International Renewable Energy Agency, 2020. Geothermal development in eastern Africa, Recommendation for power and direct use.

3. Japan international cooperation agency, 2010. Situation Analyses Study on Geothermal Development in Arica. West Japan Engineering consultants, inc.

4. Websites for the shapefile: 
- Most populated sites shapefile and administrative territorial division shapefile: https://data.humdata.org

- Electrical and roads connection shapefile: https:/learthworks.stanford.edu/catalog.html?f\% 5 Bdc format s $\% 5 D \% 5 B \% 5 D=$ Shapefile\&pag $\underline{e=11 \& q=k e n y a / / \text { https://data.worldbank.org/ }}$

- AGID country information shapefile: https://www.africageoportal.com/pages/africa-living-atlas

- Supporting shapefiles: https://open.africa

5. Solomon Kebebe, 2015. Country update on geothermal exploration and development in Ethiopia. Short Course IX on Exploration for geothermal resources organized by UNU-GPT, GDC and KenGen, Kenya.

6. MANNVIT and VERKIS, 2020. Geothermal drilling in Corbetti Ethiopia. Pre-qualification. https://www.thinkgeoenergy.com/wp-content/uploads/2020/06/Corbetti-Pre-qualification-for-DrillingContractor-Final.pdf

7. Martha Mburu, 2010. Feasibility study on direct utilisation of energy from geothermal brine - a case study of olkaria geothermal power plant, Kenya. Proceeding World Geothermal Congress, Bali.

8. Mwangi and Mburu, 2005. Geothermal energy potential in Africa. Professional lectures at United Nations University, Geothermal training programme, Iceland.

9. Hole H. and Mills T. D. 2003. Geothermal Greenhouse Heating at Oserian Farm, Lake Naivasha, Kenya", 2nd KenGen geothermal conference, Nairobi Kenya.

10. John Lagat, 2010. Direct utilization of geothermal resource in Kenya. Proceeding World Geothermal Congress, Bali.

11. R. Di Pippo. 2005. Geothermal Power Plants: Principles, Applications, case Studies and Environmental Impact. Developments and Innovation. Elsevier. 